\title{
Formal Analysis of Privacy for Routing Protocols in Mobile Ad Hoc Networks ${ }^{\star}$
}

\author{
Rémy Chrétien and Stéphanie Delaune \\ LSV, ENS Cachan \& CNRS \& INRIA Saclay Île-de-France
}

\begin{abstract}
Routing protocols aim at establishing a route between distant nodes in ad hoc networks. Secured versions of routing protocols have been proposed to provide more guarantees on the resulting routes, and some of them have been designed to protect the privacy of the users.

In this paper, we propose a framework for analysing privacy-type properties for routing protocols. We use a variant of the applied-pi calculus as our basic modelling formalism. More precisely, using the notion of equivalence between traces, we formalise three security properties related to privacy, namely indistinguishability, unlinkability, and anonymity. We study the relationship between these definitions and we illustrate them using two versions of the ANODR routing protocol.
\end{abstract}

\section{Introduction}

Mobile ad hoc networks consist of mobile wireless devices which autonomously organise their communication infrastructure. They are being used in a large array of settings, from military applications to emergency rescue; and are also believed to have future uses in e.g. vehicular networking. In such a network, each node provides the function of a router and relays packets on paths to other nodes. Finding these paths is a crucial functionality of any ad hoc network. Specific protocols, called routing protocols, are designed to ensure this functionality known as route discovery.

Since an adversary can easily paralyse the operation of a whole network by attacking the routing protocol, substantial efforts have been made to provide efficient and secure routing protocols 211418 . For instance, in order to prevent a malicious node to insert and delete nodes inside a path, cryptographic mechanisms such as encryption, signature, and MAC are used. However, there is a privacy problem related to the way routes are discovered by those routing protocols. Indeed, most routing protocols (e.g. [14[18]) flood the entire network with a route request message containing the names of the source and the destination of the intended communication. Thus, an eavesdropper can easily observe who wants to communicate with whom even if he is not on the route between the communicating nodes. Since then, in order to limit privacy issues, several anonymous routing protocols have been developed, e.g. ANODR [15], AnonDSR [20] to resist against passive adversaries showing no suspicious behaviours.

^ This work has been partially supported by the project JCJC VIP ANR-11-JS02-006.

D. Basin and J.C. Mitchell (Eds.): POST 2013, LNCS 7796, pp. 1-20, 2013.

(C) Springer-Verlag Berlin Heidelberg 2013 
Because security protocols are in general notoriously difficult to design and analyse, formal verification techniques are particularly important. For example, a flaw has been discovered in the Single-Sign-On protocol used e.g. by Google Apps 4]. It has been shown that a malicious application could very easily gain access to any other application (e.g. Gmail or Google Calendar) of their users. This flaw has been found when analyzing the protocol using formal methods, abstracting messages by a term algebra and using the AVISPA platform [5].

Whereas secrecy and authentication are well-understood notions, anonymity itself is ill-defined: behind the general concept lie distinct considerations which share the general idea of not disclosing any crucial information to an attacker on the network. Thus, formalizing privacy-type properties is not an easy task and has been the subject of several papers in the context of electronic voting (e.g. [127]), RFID systems (e.g. [39]), or anonymizing protocols (e.g. [1613]). Whereas some of them rely on a probabilitistic notion of anonymity (e.g. [19]), we focus on deterministic ones, for which formal analysis appears more natural. All these definitions share a common feature: they are based on a notion of equivalence that allows one to express the fact that two situations are similar, i.e. indistinguishable from the point of view of the attacker.

Our contributions. In this paper, we propose a formal framework for analyzing privacy-type properties in the context of routing protocols. We use a variant of the applied-pi calculus as our basic modeling formalism [1, which has the advantage of being based on well-understood concepts and to allow us to model various cryptographic primitives by the means of an equational theory (see Sections 2 and 3). However, in order to model route discovery protocols, we have to adapt it to take into account several features of those protocols, e.g. the topology of the network, broadcast communication, internal states of the nodes, etc

Then, we investigate the different properties a routing protocol could achieve to be considered indeed anonymous in presence of a passive attacker. We propose three different families of such properties: indistinguishability, which deals with the possibility to distinguish some external action undertaken by an agent from another (see Section 4); unlinkability, which is related to the ability for the attacker to link certain actions together (see Section 5); and finally anonymity which concerns the disclosure of information such as the identity of the sender, or the identity of the receiver (see Section 6). We formalise those properties using a notion of equivalence between traces. Some difficulties arise due to the application under study. In particular, to achieve those security properties, we have to ensure that the network is active enough, and thus we have to provide a formal definition of this notion. We study the relationship between these privacytype properties and we illustrate our definitions on two versions of the ANODR routing protocol [15].

Related work. Notions of privacy have been studied for RFID protocols [3] such as the key establishment protocol used in the electronic passport application. Similarly, formal definitions and proofs of anonymity for anonymizing protocols, like the onion routing, were proposed in [16 13. Nevertheless these formalisms do 
not allow one to freely specify network topologies, a crucial feature for mobile adhoc routing. Moreover, as an extension of the applied pi-calculus, our formalism is not bound to a fixed set of primitives but make our definition usable for a large class of routing protocols. A more detailed version of this paper is available in [10].

\section{Messages and Attacker Capabilities}

As often in protocol analysis, cryptographic primitives are assumed to work perfectly. However, we do not consider an active attacker who controls the entire network as generally done when analyzing more classical protocols. We will consider an eavesdropper who listens to some nodes of the network or even all of them. Basically, he can see messages that are sent from locations he is spying on, and can only encrypt, decrypt, sign messages or perform other cryptographic operations if he has the relevant keys.

\subsection{Messages}

For modeling messages, we consider an arbitrary term algebra, which provides a lot of flexibility in terms of which cryptographic primitives can be modelled. In such a setting, messages are represented by terms where cryptographic primitives such as encryption, signature, and hash function, are represented by function symbols. More precisely, we consider a signature $(\mathcal{S}, \Sigma)$ made of a set of sorts $\mathcal{S}$ and a set of function symbols $\Sigma$ together with arities of the form $\operatorname{ar}(\mathbf{f})=s_{1} \times$ $\ldots \times s_{k} \rightarrow s$ where $f \in \Sigma$, and $s, s_{1}, \ldots, s_{k} \in \mathcal{S}$. We consider an infinite set of variables $\mathcal{X}$ and an infinite set of names $\mathcal{N}$ which are used for representing keys, nonces, etc We assume that names and variables are given with sorts. Terms are defined as names, variables, and function symbols applied to other terms. Of course function symbol application must respect sorts and arities. For $\mathcal{A} \subseteq \mathcal{X} \cup \mathcal{N}$, the set of terms built from $\mathcal{A}$ by applying function symbols in $\Sigma$ is denoted by $\mathcal{T}(\Sigma, \mathcal{A})$.

We write $\operatorname{vars}(u)$ (resp. names $(u)$ ) for the set of variables (resp. names) that occur in a term $u$. A term $u$ is said to be a ground term if vars $(u)=\emptyset$. Regarding the sort system, we consider a special sort agent that only contains names and variables. These names represent the names of the agents, also called the nodes of the network. We assume a special sort msg that subsumes all the other sorts, i.e. any term is of sort msg.

For our cryptographic purposes, it is useful to distinguish a subset $\Sigma_{\text {pub }}$ of $\Sigma$, made of public symbols, i.e. the symbols made available to the attacker. A recipe is a term in $\mathcal{T}\left(\Sigma_{\text {pub }}, \mathcal{X} \cup \mathcal{N}\right)$, that is, a term containing no private (non-public) symbols. Moreover, to model algebraic properties of cryptographic primitives, we define an equational theory by a finite set $\mathrm{E}$ of equations $u=v$ with $u, v \in \mathcal{T}(\Sigma, \mathcal{X})$ (note that $u, v$ do not contain names). We define $=_{\mathrm{E}}$ to be the smallest equivalence relation on terms, that contains $E$ and that is closed under application of function symbols and substitutions of terms for variables. 
Example 1. A typical signature for representing secured routing protocols is the signature $(\mathcal{S}, \Sigma)$ defined by

$-\mathcal{S}=\{$ agent, msg $\}$, and

$-\Sigma=\left\{\langle\rangle, \operatorname{proj}_{1}\right.$, $\operatorname{proj}_{2}$, senc, sdec, aenc, adec, pub, prv, req, rep, src, dest, key $\}$

with the following arities:

$$
\begin{aligned}
& \text { senc, sdec, aenc, adec, }\langle\rangle: \mathrm{msg} \times \mathrm{msg} \rightarrow \mathrm{msg} \quad \text { pub, prv }: \text { agent } \rightarrow \text { msg } \\
& \text { req, rep, src, dest, key : } \rightarrow \text { msg } \quad \operatorname{proj}_{1}, \operatorname{proj}_{2}: \mathrm{msg} \rightarrow \mathrm{msg}
\end{aligned}
$$

The constants req and rep are used to identify the request phase and the reply phase, src, dest, and key are some other public constants. The function symbols sdec, senc (resp. adec and aenc) of arity 2 represent symmetric (resp. asymmetric) decryption and encryption. Pairing is modelled using a symbol of arity 2 , denoted \langle\rangle , and projection functions are denoted $\operatorname{proj}_{1}$ and $\operatorname{proj}_{2}$. We denote by $\operatorname{pub}(A)$ (resp. $\operatorname{prv}(A))$ the public key (resp. the private key) associated to the agent $A$. Moreover, we assume that prv $\notin \Sigma_{\text {pub }}$. Then, we consider the equational theory $\mathrm{E}$, defined by the following equations $(i \in 1,2)$ :

$\operatorname{sdec}(\operatorname{senc}(x, y), y)=x \quad \operatorname{adec}(\operatorname{aenc}(x, \operatorname{pub}(y)), \operatorname{prv}(y))=x \quad \operatorname{proj}_{i}\left(\left\langle x_{1}, x_{2}\right\rangle\right)=x_{i}$

For sake of clarity, we write $\left\langle t_{1}, t_{2}, t_{3}\right\rangle$ for the term $\left\langle t_{1},\left\langle t_{2}, t_{3}\right\rangle\right\rangle$.

Substitutions are written $\sigma=\left\{x_{1} \triangleright u_{1}, \ldots, x_{n} \triangleright u_{n}\right\}$ where its domain is written $\operatorname{dom}(\sigma)=\left\{x_{1}, \ldots, x_{n}\right\}$, and its image is written $i m g(\sigma)=\left\{u_{1}, \ldots, u_{n}\right\}$. We only consider well-sorted substitutions, that is substitutions for which $x_{i}$ and $u_{i}$ have the same sort. The application of a substitution $\sigma$ to a term $u$ is written $u \sigma$. A most general unifier of two terms $u$ and $v$ is a substitution denoted by $m g u(u, v)$. We write $m g u(u, v)=\perp$ when $u$ and $v$ are not unifiable.

\subsection{Attacker Capabilities}

To represent the knowledge of an attacker (who may have observed a sequence of messages $t_{1}, \ldots, t_{\ell}$ ), we use the concept of frame. A frame $\phi=$ new $\tilde{n} . \sigma$ consists of a finite set $\tilde{n} \subseteq \mathcal{N}$ of restricted names (those unknown to the attacker), and a substitution $\sigma$ of the form $\left\{y_{1} \triangleright t_{1}, \ldots, y_{\ell} \triangleright t_{\ell}\right\}$ where each $t_{i}$ is a ground term. The variables $y_{i}$ enable an attacker to refer to each $t_{i}$. The domain of the frame $\phi$, written $\operatorname{dom}(\phi)$, is $\operatorname{dom}(\sigma)=\left\{y_{1}, \ldots, y_{\ell}\right\}$.

In the frame $\phi=$ new $\tilde{n} . \sigma$, the names $\tilde{n}$ are bound in $\sigma$ and can be renamed. Moreover names that do not appear in $\phi$ can be added or removed from $\tilde{n}$. In particular, we can always assume that two frames share the same set of restricted names. Thus, in the definition below, we will assume w.l.o.g. that the two frames $\phi_{1}$ and $\phi_{2}$ have the same set of restricted names.

Definition 1 (static equivalence). We say that two frames $\phi_{1}=$ new $\tilde{n} . \sigma_{1}$ and $\phi_{2}=$ new $\tilde{n} . \sigma_{2}$ are statically equivalent, $\phi_{1} \sim_{\mathrm{E}} \phi_{2}$, when $\operatorname{dom}\left(\phi_{1}\right)=$ $\operatorname{dom}\left(\phi_{2}\right)$, and for all recipes $M, N$ such that names $(M, N) \cap \tilde{n}=\emptyset$, we have that: $M \sigma_{1}=\mathrm{E} N \sigma_{1}$ if, and only if, $M \sigma_{2}={ }_{\mathrm{E}} N \sigma_{2}$. 
Intuitively, two frames are equivalent when the attacker cannot see the difference between the two situations they represent, i.e., his ability to distinguish whether two recipes $M, N$ produce the same term does not depend on the frame.

Example 2. Let $\phi_{\text {req }}=\operatorname{new} n .\left\{y_{1} \triangleright \operatorname{senc}(\langle\right.$ req, $\left.n\rangle, k)\right\}$ and $\phi_{\text {rep }}=$ new $n .\left\{y_{1} \triangleright\right.$ $\operatorname{senc}(\langle\operatorname{rep}, n\rangle, k)\}$ be two frames. Considering the equational theory $\mathrm{E}$ introduced in Example 1, we have that $\phi_{\text {req }} \chi_{\mathrm{E}} \phi_{\text {rep }}$ since the recipes $M=\operatorname{proj}_{1}\left(\operatorname{sdec}\left(y_{1}, k\right)\right)$ and $N=$ req allow one to distinguish the two frames. However, we have that new $k . \phi_{\text {req }} \sim_{\mathrm{E}}$ new $k . \phi_{\text {rep }}$. Indeed, without knowing the key $k$, the attacker is unable to observe the differences between the two messages. This is a non-trivial equivalence that can be established using an automatic tool (e.g. ProVerif [8]).

\section{Models for Protocols}

In this section, we introduce the cryptographic process calculus that we will use for describing protocols. Several well-studied calculi already exist to analyse security protocols and privacy-type properties (e.g. 21]). However, modelling ad-hoc routing protocols requires several additional features. Our calculus is actually inspired from some other calculi (e.g. [17/6 11]) which allow mobile wireless networks and their security properties to be formally described and analysed. We adapt those formalisms in order to be able to express privacy-type properties such as those studied in this paper.

\subsection{Syntax}

The intended behavior of each node of the network can be modelled by a process defined by the grammar given below ( $u$ is a term that may contain variables, $n$ is a name, and $\Phi$ is a formula). Our calculus is parametrized by a set $\mathcal{L}$ of formulas whose purpose is to represent various tests performed by the agents (e.g. equality tests, neighbourhood tests). We left this set unspecified since it is not relevant for this work. For illustration purposes, we only assume that the set $\mathcal{L}$ contains at least equality and disequality tests.

$\begin{aligned} P, Q:= & 0 & & \text { null process } \\ & \operatorname{in}(u) \cdot P & & \text { reception } \\ & \text { out }(u) \cdot P & & \text { emission } \\ & \text { if } \Phi \text { then } P \text { else } Q & & \text { conditional } \Phi \in \mathcal{L} \\ & \text { store }(u) . P & & \text { storage } \\ & \text { read } u[\Phi] \text { then } P \text { else } Q & & \text { reading } \\ & P \mid Q & & \text { parallel composition } \\ & ! P & & \text { replication } \\ & \text { new } n . P & & \text { fresh name generation }\end{aligned}$

The process "in $(u) . P$ " expects a message $m$ of the form $u$ and then behaves like $P \sigma$ where $\sigma$ is such that $m=u \sigma$. The process "out $(u) . P$ " emits $u$, and then behaves like $P$. The variables that occur in $u$ will be instantiated when the 
evaluation will take place. The process store $(u) . P$ stores $u$ in its storage list and then behaves like $P$. The process read $u[\Phi]$ then $P$ else $Q$ looks for a message of the form $u$ that satisfies $\Phi$ in its storage list and then, if such an element $m$ is found, it behaves like $P \sigma$ where $\sigma$ is such that $m=u \sigma$. Otherwise, it behaves like $Q$. The other operators are standard.

Sometimes, for the sake of clarity, we will omit the null process. We also omit the else part when $Q=0$. We write fvars $(P)$ for the set of free variables that occur in $P$, i.e. the set of variables that are not in the scope of an input or a read. We consider ground processes, i.e. processes $P$ such that fvars $(P)=\emptyset$, and parametrized processes, denoted $P\left(z_{1}, \ldots, z_{n}\right)$ where $z_{1}, \ldots, z_{n}$ are variables of sort agent, and such that fvars $(P) \subseteq\left\{z_{1}, \ldots, z_{n}\right\}$. A routing protocol is a set of parametrized processes.

\subsection{Example: ANODR}

ANODR is an anonymous on-demand routing protocol that has been designed to prevent traffic analysis in ad hoc networks [15]. We consider a simplified version of this protocol, denoted $\mathcal{P}_{\text {ANODR }}^{\text {simp }}$. For sake of readability, we give below an Alice and Bob version of this two-phase protocol where we omit some $\langle\cdots, \cdot\rangle$ and we use $\{\cdot\}$. instead of senc and aenc.

$$
\begin{aligned}
& S \rightarrow V_{1}:\left\langle\text { req, } i d,\{D, \text { chall }\}_{\operatorname{pub}(D)},\{S, \operatorname{src}\}_{k_{S}}\right\rangle \\
& V_{1} \rightarrow V_{2}:\left\langle\text { req, id, }\{D, \text { chall }\}_{\text {pub }(D)},\left\{V_{1},\{S, \text { src }\}_{k_{S}}\right\}_{k_{1}}\right\rangle \\
& V_{2} \rightarrow D:\left\langle\text { req, id, }\{D, \text { chall }\}_{\text {pub }(D)},\left\{V_{2},\left\{V_{1},\{S, \operatorname{src}\}_{k_{S}}\right\}_{k_{1}}\right\}_{k_{2}}\right\rangle \\
& D \rightarrow V_{2}:\left\langle\text { rep, } N_{D} \text {, chall, }\left\{V_{2},\left\{V_{1},\{S, \text { src }\}_{k_{S}}\right\}_{k_{1}}\right\}_{k_{2}}\right\rangle \\
& V_{2} \rightarrow V_{1}:\left\langle\text { rep, } N_{2} \text {, chall, }\left\{V_{1},\{S, \text { src }\}_{k_{S}}\right\}_{k_{1}}\right\rangle \\
& V_{1} \rightarrow S:\left\langle\text { rep, } N_{1} \text {, chall, }\{S, \text { src }\}_{k_{S}}\right\rangle
\end{aligned}
$$

Request phase. The source initiates route discovery by locally broadcasting a request. The constant req is used to identify the request phase whereas $i d$ is an identifier of the request. The third component of the request is a cryptographic trapdoor that can only be opened by the destination; and the last one is a cryptographic onion that is used for route establishment. At this stage, the onion built by the source contains only one layer.

Then, intermediate nodes relay the request over the network, except if they have already seen it. However, contrary to what happen in many routing protocols, the names of the intermediate nodes are not accumulated in the route request packet. This is important to prevent traffic analysis.

Reply phase. When the destination $D$ receives the request, it opens the trapdoor and builds a route reply.

During the reply phase, the message travels along the route back to $S$. The intermediary node decrypt the onion using its own key which has been generated during the request phase. If its own identity does not match the first field of the decrypted result, it then discards the packet. Otherwise, the node is on the anonymous route. It generates a random number (namely $N_{D}, N_{1}$, or $N_{2}$ ), stores 
the correspondence between the nonce it receives and the one it has generated. It peels off one layer of the onion, replaces the nonce with its own nonce, and then locally broadcasts the reply packet.

Formally, this protocol is composed of four parametrized processes that can be modelled using the signature given in Example 1 Let $i d$ be a name, $z_{S}, z_{V}, z_{D}$ be variables of sort agent, and $x_{N}, x_{i d}, x_{t r}$ and $x_{\text {onion }}$ be variables of sort msg.

The process executed by the agent $z_{S}$ initiating the search of a route towards a node $z_{D}$ is:

$$
\begin{gathered}
P_{\text {src }}\left(z_{S}, z_{D}\right)=\text { new } i d \text {.new chall.new } k_{S} \text {.out }\left(u_{1}\right) \cdot \operatorname{in}\left(u_{2}\right) \cdot \operatorname{store}\left(\left\langle z_{D}, x_{N}\right\rangle\right) \\
\text { where }\left\{\begin{array}{l}
u_{1}=\left\langle\text { req, } i d, \text { aenc }\left(\left\langle z_{D}, \text { chall }\right\rangle, \operatorname{pub}\left(z_{D}\right)\right), \operatorname{senc}\left(\left\langle z_{S}, \operatorname{src}\right\rangle, k_{S}\right)\right\rangle \\
u_{2}=\left\langle\text { rep }, x_{N}, \text { chall }, \operatorname{senc}\left(\left\langle z_{S}, \operatorname{src}\right\rangle, k_{S}\right)\right\rangle
\end{array}\right.
\end{gathered}
$$

The source $z_{S}$ builds a request message and sends it. Then, the source is waiting for a reply containing the same cryptographic onion as the one used in the request, a proof of global trapdoor opening (here modelled as a nonce chall), and a locally unique random route pseudonym $N$. Lastly, the source will store that destination $D$ can be reached using the route pseudonym $N$ as the next hop.

The process executed by an intermediary node $z_{V}$ during the request phase is described below. For sake of simplicity, we did not model the fact that a duplicated request message is directly discarded.

$$
P_{\text {int }}^{\text {req }}\left(z_{V}\right)=\operatorname{in}\left(w_{1}\right) \text {.if } \neg \Phi_{\text {req }} \text { then }\left(\text { new } k_{V} \text {.store }\left(\left\langle\text { key, } k_{V}\right\rangle\right) \text {.out }\left(w_{2}\right)\right)
$$

where $\left\{\begin{array}{l}w_{1}=\left\langle\text { req }, x_{i d}, x_{t r}, x_{\text {onion }}\right\rangle \quad \Phi_{\text {req }}=\operatorname{proj}_{1}\left(\operatorname{adec}\left(x_{t r}, \operatorname{prv}\left(z_{V}\right)\right)\right)=z_{V} \\ w_{2}=\left\langle\text { req }, x_{i d}, x_{t r}, \operatorname{senc}\left(\left\langle z_{V}, x_{\text {onion }}\right\rangle, k_{V}\right)\right\rangle\end{array}\right.$

The process executed by the destination node $z_{D}$ is the following:

$$
P_{\text {dest }}\left(z_{D}\right)=\operatorname{in}\left(v_{1}\right) . \text { if } \Phi_{\text {dest }} \text { then }\left(\text { new } N \text {.out }\left(v_{2}\right)\right)
$$

where $\left\{\begin{array}{l}v_{1}=\left\langle\text { req }, x_{i d}, x_{t r}, x_{\text {onion }}\right\rangle \quad \Phi_{\text {dest }}=\operatorname{proj}_{1}\left(\operatorname{adec}\left(x_{t r}, \operatorname{prv}\left(z_{D}\right)\right)\right)=z_{D} \\ v_{2}=\left\langle\operatorname{rep}, N, \operatorname{proj}_{2}\left(\operatorname{adec}\left(x_{t r}, \operatorname{prv}\left(z_{D}\right)\right)\right), x_{\text {onion }}\right\rangle\end{array}\right.$

The process executed by an intermediary node $z_{V}$ during the reply phase is as follows:

$$
P_{\text {int }}^{\text {rep }}\left(z_{V}\right)=\operatorname{in}\left(w_{1}^{\prime}\right) \text {.read }\langle\text { key, } y\rangle\left[\Phi_{\text {rep }}\right] \text { then }\left(\text { new } N^{\prime} \text {.store }\left(\left\langle x_{N}, N^{\prime}\right\rangle\right) \text {.out }\left(w_{2}^{\prime}\right)\right)
$$

where $\left\{\begin{array}{l}w_{1}^{\prime}=\left\langle\operatorname{rep}, x_{N}, x_{p r}, x_{\text {onion }}\right\rangle \quad \Phi_{\text {rep }}=\operatorname{proj}_{1}\left(\operatorname{sdec}\left(x_{\text {onion }}, y\right)\right)=z_{V} \\ w_{2}^{\prime}=\left\langle\operatorname{rep}, N^{\prime}, x_{p r}, \operatorname{proj}_{2}\left(\operatorname{sdec}\left(x_{\text {onion }}, y\right)\right)\right\rangle\end{array}\right.$

Once, a route between $S$ and $D$ has been established using this protocol, a data packet can then be sent from $S$ to $D$ using the route pseudonyms that nodes have stored in their storage list. 


\subsection{Configuration and Topology}

Each process is located at a node of the network, and we consider an eavesdropper who observes messages sent from particular nodes. More precisely, we assume that the topology of the network is represented by a pair $\mathcal{T}=(G, \mathcal{M})$ where:

- $G=(V, E)$ is an undirected finite graph with $V \subseteq\{A \in \mathcal{N} \mid A$ of sort agent $\}$, where an edge in the graph models the fact that two agents are neighbors.

$-\mathcal{M}$ is a set of nodes, the malicious nodes, from which the attacker is able to listen to their outputs.

We consider several malicious nodes, and our setting allows us to deal with the case of a global eavesdropper (i.e. $\mathcal{M}=V$ ). A trivial topology is a topology $\mathcal{T}=(G, \mathcal{M})$ with $\mathcal{M}=\emptyset$.

A configuration of the network is a quadruplet $(\mathcal{E} ; \mathcal{P} ; \mathcal{S} ; \sigma)$ where:

$-\mathcal{E}$ is a finite set of names that represents the names restricted in $\mathcal{P}, \mathcal{S}$ and $\sigma$;

- $\mathcal{P}$ is a multiset of expressions of the form $\lfloor P\rfloor_{A}$ that represents the process $P$ executed by the agent $A \in V$. We write $\lfloor P\rfloor_{A} \cup \mathcal{P}$ instead of $\left\{\lfloor P\rfloor_{A}\right\} \cup \mathcal{P}$.

- $\mathcal{S}$ is a set of expressions of the form $\lfloor u\rfloor_{A}$ with $A \in V$ and $u$ a ground term. $\lfloor u\rfloor_{A}$ represents the term $u$ stored by the agent $A \in V$.

- $\sigma=\left\{y_{1} \triangleright u_{1}, \ldots, y_{n} \triangleright u_{n}\right\}$ where $u_{1}, \ldots, u_{n}$ are ground terms (the messages observed by the attacker), and $y_{1}, \ldots, y_{n}$ are variables.

\subsection{Execution Model}

Each node broadcasts its messages to all its neighbors. The communication system is formally defined by the rules of Figure 1 They are parametrized by the underlying topology $\mathcal{T}$. The Comm rule allows nodes to communicate provided they are (directly) connected in the underlying graph, without the attacker actively interfering. We do not assume that messages are necessarily delivered to the intended recipients. They may be lost. The exchange message is learnt by the attacker as soon as the node that emits it is under its scrutiny.

The other rules are quite standard.

We write $\rightarrow$ instead of $\rightarrow \mathcal{T}$ when the underlying network topology $\mathcal{T}$ is clear from the context. Let $\mathcal{A}$ be the alphabet of actions where the special symbol $\tau \in \mathcal{A}$ represents an unobservable action. For every $\ell \in \mathcal{A}$, the relation $\stackrel{\ell}{\rightarrow}$ has been defined in Figure 1. For every $w \in \mathcal{A}^{*}$ the relation $\stackrel{w}{\rightarrow}$ on configurations is defined in the usual way. By convention $K \stackrel{\epsilon}{\rightarrow} K$ where $\epsilon$ denotes the empty word. For every $s \in(\mathcal{A} \backslash\{\tau\})^{*}$, the relation $\stackrel{s}{\Longrightarrow}$ on configurations is defined by: $K \stackrel{s}{\Longrightarrow} K^{\prime}$ if, and only if, there exists $w \in \mathcal{A}^{*}$ such that $K \stackrel{w}{\rightarrow} K^{\prime}$ and $s$ is obtained from $w$ by erasing all occurrences of $\tau$. Intuitively, $K \stackrel{s}{\Rightarrow} K^{\prime}$ means that $K$ transforms into $K^{\prime}$ by experiment $s$.

An initial configuration associated to a topology $\mathcal{T}=(G, \mathcal{M})$ and a routing

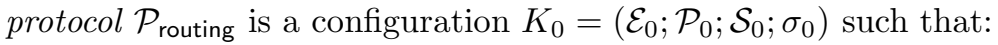

$$
\mathcal{P}_{0}=\bigcup_{\substack{P \in \mathcal{P}_{\text {routing }} \\ A, B_{1}, \ldots, B_{k} \in V}}\left\lfloor! P\left(A, B_{1}, \ldots, B_{k}\right)\right\rfloor_{A} .
$$


$\operatorname{Comm}\left(\mathcal{E} ;\lfloor\text { out }(t) . P\rfloor_{A} \cup\left\{\left\lfloor\text { in }\left(u_{j}\right) . P_{j}\right\rfloor_{A_{j}} \mid m g u\left(t, u_{j}\right) \neq \perp \wedge\left(A, A_{j}\right) \in E\right\} \cup \mathcal{P} ; \mathcal{S} ; \sigma\right)$

where $\left\{\begin{array}{l}\sigma_{j}=m g u\left(t, u_{j}\right) \\ \sigma^{\prime}=\sigma \cup\{y \triangleright t\} \text { where } y \text { is a fresh variable and } \ell=(\operatorname{out}(y), A) \text { if } A \in \mathcal{M} ; \\ \sigma^{\prime}=\sigma \text { and } \ell=\tau \text { otherwise }\end{array}\right.$

$$
\stackrel{\ell}{\rightarrow} \mathcal{T}\left(\mathcal{E} ;\left\{\left\lfloor P_{j} \sigma_{j}\right\rfloor_{A_{j}}\right\} \cup\lfloor P\rfloor_{A} \cup \mathcal{P} ; \mathcal{S} ; \sigma^{\prime}\right)
$$

STORE

$$
\left(\mathcal{E} ;\lfloor\text { store }(t) . P\rfloor_{A} \cup \mathcal{P} ; \mathcal{S} ; \sigma\right) \stackrel{\tau}{\rightarrow} \mathcal{T}\left(\mathcal{E} ;\lfloor P\rfloor_{A} \cup \mathcal{P} ;\lfloor t\rfloor_{A} \cup \mathcal{S} ; \sigma\right)
$$

$\operatorname{ReAd-Then~}\left(\mathcal{E} ;\lfloor\operatorname{read} u[\Phi] \text { then } P \text { else } Q\rfloor_{A} \cup \mathcal{P} ;\lfloor t\rfloor_{A} \cup \mathcal{S} ; \sigma\right)$

$$
\stackrel{\tau}{\rightarrow} \mathcal{T}\left(\mathcal{E} ;\lfloor P \lambda\rfloor_{A} \cup \mathcal{P} ;\lfloor t\rfloor_{A} \cup \mathcal{S} ; \sigma\right)
$$

when $\lambda=m g u(t, u)$ exists and $\Phi \lambda$ is evaluated to true

$\operatorname{ReAD-ELSE}\left(\mathcal{E} ;\lfloor\operatorname{read} u[\Phi] \text { then } P \text { else } Q\rfloor_{A} \cup \mathcal{P} ; \mathcal{S} ; \sigma\right)$

$$
\stackrel{\tau}{\rightarrow} \mathcal{T}\left(\mathcal{E} ;\lfloor Q\rfloor_{A} \cup \mathcal{P} ; \mathcal{S} ; \sigma\right)
$$

if for all $t$ such that $\lfloor t\rfloor_{A} \in \mathcal{S}, m g u(t, u)=\perp$ or $\Phi m g u(t, u)$ is evaluated to false

If-Then $\quad\left(\mathcal{E} ;\lfloor\text { if } \Phi \text { then } P \text { else } Q\rfloor_{A} \cup \mathcal{P} ; \mathcal{S} ; \sigma\right) \stackrel{\tau}{\rightarrow} \mathcal{T}\left(\mathcal{E} ;\lfloor P\rfloor_{A} \cup \mathcal{P} ; \mathcal{S} ; \sigma\right)$

if $\Phi$ is evaluated to true

If-ELSE $\quad\left(\mathcal{E} ;\lfloor\text { if } \Phi \text { then } P \text { else } Q\rfloor_{A} \cup \mathcal{P} ; \mathcal{S} ; \sigma\right) \stackrel{\tau}{\rightarrow} \mathcal{T}\left(\mathcal{E} ;\lfloor Q\rfloor_{A} \cup \mathcal{P} ; \mathcal{S} ; \sigma\right)$

if $\Phi$ is evaluated to false

PAR

$\left(\mathcal{E} ;\left\lfloor P_{1} \mid P_{2}\right\rfloor_{A} \cup \mathcal{P} ; \mathcal{S} ; \sigma\right) \stackrel{\tau}{\rightarrow} \mathcal{T}\left(\mathcal{E} ;\left\lfloor P_{1}\right\rfloor_{A} \cup\left\lfloor P_{2}\right\rfloor_{A} \cup \mathcal{P} ; \mathcal{S} ; \sigma\right)$

REPL

$\left(\mathcal{E} ;\lfloor! P\rfloor_{A} \cup \mathcal{P} ; \mathcal{S} ; \sigma\right) \stackrel{\tau}{\rightarrow} \mathcal{T}\left(\mathcal{E} ;\lfloor P\rfloor_{A} \cup\lfloor! P\rfloor_{A} \cup \mathcal{P} ; \mathcal{S} ; \sigma\right)$

NEW

$\left(\mathcal{E} ;\lfloor\text { new } n . P\rfloor_{A} \cup \mathcal{P} ; \mathcal{S} ; \sigma\right) \stackrel{\tau}{\rightarrow} \mathcal{T}\left(\mathcal{E} \cup\left\{n^{\prime}\right\} ;\left\lfloor P\left\{n^{\prime} / n\right\}\right\rfloor_{A} \cup \mathcal{P} ; \mathcal{S} ; \sigma\right)$ where $n^{\prime}$ is a fresh name

Fig. 1. Transition system

Such a configuration represents the fact that each node can play any role of the protocol an unbounded number of times. Moreover, the agent who executes the process is located at the right place. A typical initial configuration will consist of $\mathcal{E}_{0}=\mathcal{S}_{0}=\sigma_{0}=\emptyset$, but depending on the protocol under study, we may want to populate the storage lists of some nodes.

Example 3. Let $\mathcal{T}_{0}=\left(G_{0}, \mathcal{M}_{0}\right)$ be a topology where $G_{0}$ is described below, and consider a global eavesdropper, i.e. $\mathcal{M}_{0}=\{A, B, C, D\}$.

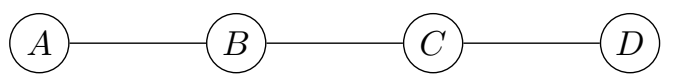

We consider the execution of the protocol $\mathcal{P}_{\text {ANODR }}^{\text {simp }}$ where $B$ acts as a source to obtain a route to $D$. Receiving this request, and not being the destination, its neighbour $C$ acts as a request forwarding node. We have that:

$$
\operatorname{tr}=K_{0} \stackrel{\tau}{\rightarrow} \stackrel{\tau}{\rightarrow} \stackrel{\tau}{\rightarrow} \stackrel{\text { out }\left(y_{1}\right), B}{\longrightarrow}\left(\mathcal{E}_{1} ; \mathcal{P}_{1} ; \mathcal{S}_{1} ; \sigma_{1}\right) \stackrel{\tau}{\rightarrow} \stackrel{\tau}{\rightarrow} \stackrel{\tau}{\rightarrow} \stackrel{\text { out }\left(y_{2}\right), C}{\longrightarrow}\left(\mathcal{E}_{2} ; \mathcal{P}_{2} ; \mathcal{S}_{2} ; \sigma_{2}\right)
$$


where: $\begin{cases}K_{0}=\left(\emptyset ; \mathcal{P}_{0} ; \emptyset ; \emptyset\right) \text { initial configuration associated to } \mathcal{T}_{0} \text { and } \mathcal{P}_{\text {ANODR }}^{\text {simp }} . \\ \mathcal{E}_{1}=\left\{i d, \text { chall, } k_{B}\right\} & \mathcal{E}_{2}=\left\{i d, \text { chall }, k_{B}, k_{C}\right\} \\ \mathcal{S}_{1}=\emptyset & \mathcal{S}_{2}=\left\{\left\lfloor\left\langle\text { key }, k_{C}\right\rangle\right\rfloor C\right\} \\ \sigma_{1}=\left\{y_{1} \triangleright u\right\} & \sigma_{2}=\left\{y_{1} \triangleright u, y_{2} \triangleright v\right\} \\ u=\left\langle\text { req, } i d, \operatorname{aenc}(\langle D, \text { chall }\rangle, \operatorname{pub}(D)), \operatorname{senc}\left(\langle B, \operatorname{src}\rangle, k_{B}\right)\right\rangle \\ v=\left\langle\text { req, } i d, \operatorname{aenc}(\langle D, \text { chall }\rangle, \operatorname{pub}(D)), \operatorname{senc}\left(\left\langle C, \operatorname{senc}\left(\langle B, \operatorname{src}\rangle, k_{B}\right)\right\rangle, k_{C}\right)\right\rangle\end{cases}$

The process $\left\lfloor P_{\mathrm{src}}(B, D)\right\rfloor_{B}$ that occurs in $K_{0}$ will first follow the rule NEw three times to generate the nonces $i d$, chall and $k_{B}$ leading to a new set of restricted names $\mathcal{E}_{1}$. The rule Comm is then applied between nodes $B$ and $C$. As $B \in \mathcal{M}_{0}$, the message is included in $\sigma_{1}$ to represent the knowledge gained by the attacker. As the node $C$ is not the destination, $\left\lfloor P_{\text {int }}^{\text {req }}(C)\right\rfloor_{C}$ can evolve (rule If-Then). It generates a key (rule NEw) added in $\mathcal{E}_{2}$, and stores it in $\mathcal{S}_{2}$ (rule STORE) and finally it uses Comm to broadcast the resulting message, which is also added to current substitution $\sigma_{2}$. Actually, in case we are only interested by the visible actions, this trace tr could also be written as follows:

$$
\operatorname{tr}=K_{0} \stackrel{\text { out }\left(y_{1}\right), B}{=}\left(\mathcal{E}_{1} ; \mathcal{P}_{1} ; \mathcal{S}_{1} ; \sigma_{1}\right) \stackrel{\text { out }\left(y_{2}\right), C}{=}\left(\mathcal{E}_{2} ; \mathcal{P}_{2} ; \mathcal{S}_{2} ; \sigma_{2}\right) \text {. }
$$

\subsection{Extension and Equivalence of Traces}

We cannot expect that privacy-type properties hold in any situation. We have to ensure that the traffic is sufficient. For this we need to introduce the notion of extension of a trace. Roughly, we say that a trace $\mathrm{tr}^{+}$is an extension of a trace $\mathrm{tr}_{\mathrm{if}} \mathrm{tr}^{+}$contains at least all the actions that are exhibited in $\mathrm{tr}$. In order to track of the actions, we consider annotated traces. This need comes from the fact that our calculus (and many others cryptographic calculi) does not provide us with information that allow us to retrieve who performed a given action.

We will denote $K \underset{A, \mathrm{R}}{\stackrel{\tau}{\longrightarrow}} K^{\prime}$ (resp. $K \underset{A, \mathrm{R}}{\stackrel{\text { out }(y), A}{\longrightarrow}} K^{\prime}$ ) instead of $K \stackrel{\tau}{\rightarrow} K^{\prime}$ (resp. $\left.K \stackrel{\text { out }(y), A}{\longrightarrow} K^{\prime}\right)$ to explicit the annotations. We have that $A \in V$ and $\mathrm{R}$ is a constant. Intuitively $A$ is the node that performs the action (resp. the output) whereas $\mathrm{R}$ is a constant that represents the role who is responsible of this action (resp. output). Thus, to formalise this notion of annotated trace, we associate a constant to each parametrized process part of the routing protocol under study. Theses annotations are nonetheless invisible to the attacker: she has only access to the labels of the transitions defined in our semantics. Annotations are meant to be used to specify privacy properties.

Example 4. Going back to our running example, $\mathcal{P}_{\text {ANODR }}^{\text {simp }}$ is made up of 4 roles and we associate a constant to each of them, namely Src, Req, Dest, and Rep. The annotated version of the trace $\operatorname{tr}$ described in Example 3 is:

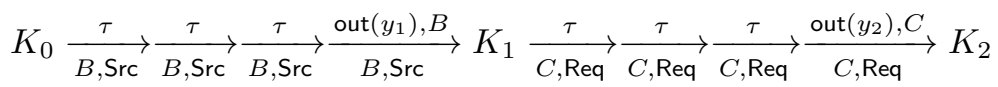

with $K_{1}=\left(\mathcal{E}_{1} ; \mathcal{P}_{1} ; \mathcal{S}_{1} ; \sigma_{1}\right)$ and $K_{2}=\left(\mathcal{E}_{2} ; \mathcal{P}_{2} ; \mathcal{S}_{2} ; \sigma_{2}\right)$. 
Given two configurations $K=(\mathcal{E} ; \mathcal{P} ; \mathcal{S} ; \sigma)$ and $K^{+}=\left(\mathcal{E}^{+} ; \mathcal{P}^{+} ; \mathcal{S}^{+} ; \sigma^{+}\right)$, we write $K \subseteq K^{+}$if $\mathcal{E} \subseteq \mathcal{E}^{+}, \mathcal{P} \subseteq \mathcal{P}^{+}, \mathcal{S} \subseteq \mathcal{S}^{+}$, and $\sigma_{\mid \operatorname{dom}(\sigma)}^{+}=\sigma$.

Definition 2 (extension of a trace). Let $\operatorname{tr}^{+}$be an annotated trace:

$$
\mathrm{tr}^{+}=K_{0} \underset{A_{1}, \mathrm{R}_{1}}{\stackrel{\ell_{1}}{\longrightarrow}} K_{1}^{+} \underset{A_{2}, \mathrm{R}_{2}}{\stackrel{\ell_{2}}{\longrightarrow}} \ldots \frac{\ell_{n}}{\underset{A_{n}, \mathrm{R}_{n}}{\longrightarrow}} K_{n}^{+} .
$$

We say that $\mathrm{tr}^{+}$is an extension of $\mathrm{tr}$, denoted $\mathrm{tr} \preccurlyeq \mathrm{tr}^{+}$, if

$$
\operatorname{tr}=K_{0} \underset{A_{k_{1}}, \mathrm{R}_{k_{1}}}{\stackrel{\ell_{k_{1}}}{\longrightarrow}} K_{k_{1}} \underset{A_{k_{2}}, \mathrm{R}_{k_{2}}}{\stackrel{\ell_{k_{2}}}{\longrightarrow}} \ldots \frac{\ell_{k_{\ell}}}{A_{k_{\ell}}, \mathrm{R}_{k_{\ell}}} K_{k_{\ell}}
$$

where $0<k_{1}<k_{2}<\ldots<k_{\ell} \leq n$, and $K_{k_{i}} \subseteq K_{k_{i}}^{+}$for each $i \in\{1, \ldots, \ell\}$.

Given an indice $i$ corresponding to an action in $\operatorname{tr}(1 \leq i \leq \ell)$, we denote by $\operatorname{ind}_{i}\left(\operatorname{tr}, \operatorname{tr}^{+}\right)$the indice of the corresponding action in $\operatorname{tr}^{+}$, i.e. $\operatorname{ind}_{i}\left(\operatorname{tr}, \operatorname{tr}^{+}\right)=k_{i}$.

Example 5. An extension of the trace tr described in Example 3 could be to let $A$ initiate a new session before $B$ tries to discover a route to $D$. Such an execution is formalised by the $\operatorname{trace} \mathrm{tr}^{+}$written below:

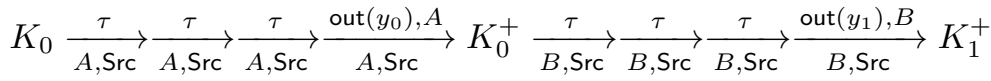

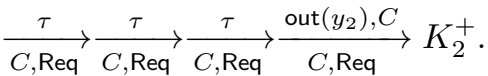

where the configurations are not detailed, but $\left(\mathcal{E}_{i} ; \mathcal{P}_{i} ; \mathcal{S}_{i} ; \sigma_{i}\right) \subseteq K_{i}^{+}(i \in\{1,2\})$.

Privacy-type security properties are often formalised using a notion equivalence (see e.g. [1239]). Here, we consider the notion of equivalence between two traces.

Definition 3 (equivalence of two traces). Let $\operatorname{tr}_{1}=K_{1} \stackrel{s_{1}}{\Longrightarrow}\left(\mathcal{E}_{1} ; \mathcal{P}_{1} ; \mathcal{S}_{1} ; \sigma_{1}\right)$ and $\operatorname{tr}_{2}=K_{2} \stackrel{s_{2}}{\Longrightarrow}\left(\mathcal{E}_{2} ; \mathcal{P}_{2} ; \mathcal{S}_{2} ; \sigma_{2}\right)$ be two traces. They are equivalent, denoted $\operatorname{tr}_{1} \approx_{\mathrm{E}} \operatorname{tr}_{2}$, if $s_{1}=s_{2}$ and new $\mathcal{E}_{1} . \sigma_{1} \sim_{\mathrm{E}}$ new $\mathcal{E}_{2} . \sigma_{2}$.

Note that only observable actions are taken into account in the definition of equivalence between two traces. Roughly, two traces are equivalent if they process the same sequence of visible outputs. The two sequences may differ (we do not require the equality between $\sigma_{1}$ and $\sigma_{2}$ ) but they should be indistinguishable from the point of view of the attacker.

Example 6. In the execution $\mathrm{tr}^{+}$provided in Example 5 one could hope to hide the fact that the node $B$ is initiating a route discovery and let the attacker think $A$ is the actual source. Let $\operatorname{tr}^{\prime}$ be the execution below where $A$ initiates a route discovery towards $D$, while nodes $B$ and $C$ act as forwarders.

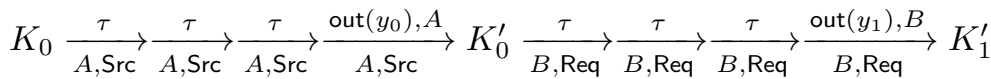

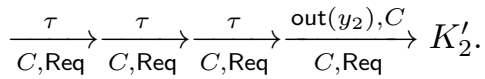

where the configurations are not detailed.

Unfortunately the attacker is able to tell the difference between $\operatorname{tr}^{+}$and $\operatorname{tr}^{\prime}$. Indeed, we have $\operatorname{tr}^{+} \nsim_{\mathrm{E}} \operatorname{tr}^{\prime}$ since the test $\operatorname{proj}_{2}\left(\operatorname{proj}_{1}\left(y_{0}\right)\right) \stackrel{?}{=} \operatorname{proj}_{2}\left(\operatorname{proj}_{1}\left(y_{1}\right)\right)$ can 
be used to distinguish the two traces. The equality test will hold in $\operatorname{tr}^{\prime}$ and not in $\mathrm{tr}^{+}$. Note that, as the annotations are invisible to the attacker, she cannot know a priori that $B$ is playing a forwarder in $\mathrm{tr}^{\prime}$.

\section{Indistinguishability}

Intuitively, indistinguishability deals with the ability for the attacker to distinguish a specific action from another. For a routing protocol such actions take the form of the various roles of the protocol. In particular we could hope, in an execution of the protocol, to make actions of the initiator or recipient indistinguishable from actions of forwarding nodes. Our definition of indistinguishability, and later of other privacy properties, depends on the network topology we are considering. Incidentally, when designing anonymous protocols, these properties should hold for large enough classes of topologies.

\subsection{Formalizing Indistinguishability}

Let Roles be a set of roles for which indistinguishability has to be preserved. A very naive definition would be to ensure that for any annotated trace $\mathrm{tr}$ issued from $K_{0}$ (the initial configuration associated to the protocol under study) where at some position $i$ the role $\mathrm{R} \in$ Roles is played and observed by the attacker, there exists an equivalent annotated trace $\operatorname{tr}^{\prime}$ where the role played at position $i$ is not in the set Roles. However, without appropriate traffic on the network, this definition is far too strong. Indeed, as soon as the source role is the only role able to spontaneously start a session, we will have no hope to achieve indistinguishability.

Definition 4 (indistinguishability). Let $K_{0}$ be an initial configuration associated to a routing protocol and a topology, and Roles be a set of roles. We say that $K_{0}$ preserves indistinguishability w.r.t. Roles if for any annotated trace tr

$$
\operatorname{tr}=K_{0} \underset{A_{1}, \mathrm{R}_{1}}{\stackrel{\ell_{1}}{\longrightarrow}} K_{1} \underset{A_{2}, \mathrm{R}_{2}}{\stackrel{\ell_{2}}{\longrightarrow}} \ldots \frac{\ell_{n}}{\underset{A_{n}, \mathrm{R}_{n}}{\longrightarrow}} K_{n}=(\mathcal{E} ; \mathcal{P} ; \mathcal{S} ; \sigma)
$$

and for any $i \in\{1, \ldots, n\}$ such that $\mathrm{R}_{i} \in$ Roles and $\ell_{i} \neq \tau$ (i.e. $\ell_{i}$ is an action observed by the attacker), there exist two annotated traces $\operatorname{tr}^{+}$and $\operatorname{tr}^{\prime}$ such that: $\mathrm{tr} \preccurlyeq \mathrm{tr}^{+}, \mathrm{tr}^{+} \approx \mathrm{tr}^{\prime}$, and $\mathrm{R}_{\text {ind }_{i}\left(\mathrm{tr}^{\prime} \mathrm{tr}^{+}\right)} \notin$ Roles where

$$
\operatorname{tr}^{\prime}=K_{0}^{\prime} \underset{A_{1}^{\prime}, \mathrm{R}_{1}^{\prime}}{\stackrel{\ell_{1}^{\prime}}{\longrightarrow}} K_{1}^{\prime} \underset{A_{2}^{\prime}, \mathrm{R}_{2}^{\prime}}{\stackrel{\ell_{2}^{\prime}}{\longrightarrow}} K_{2}^{\prime} \ldots \frac{\ell_{n^{\prime}}^{\prime}}{A_{n^{\prime}}^{\prime}, \mathrm{R}_{n^{\prime}}^{\prime}} K_{n^{\prime}}^{\prime} .
$$

The trace $\mathrm{tr}^{+}$enables us to deal with the aforementioned traffic needed to aim at preserving indistinguishability. Indeed rather than imposing the equivalence of $\operatorname{tr}$ with another trace, indistinguishability will be achieved if there exist two other traces $\mathrm{tr}^{+}$and $\mathrm{tr}^{\prime}$ which look the same to the attacker, and in which the action of interest is played by a different role. 


\subsection{Analysis of ANODR}

Now, we apply our formalisation of indistinguishability to the ANODR protocol.

Proposition 1. Let $\mathcal{T}$ be a topology with a malicious node that has only malicious neighbours, and $K_{0}$ be an initial configuration associated to $\mathcal{P}_{\text {ANODR }}^{\text {simp }}$ and $\mathcal{T}$. We have that $K_{0}$ does not preserve indistinguishability w.r.t. Src (resp. Dest).

Indeed, given a node $A$ which is, together with its neighbors, under the scrutiny of the attacker, consider a situation, i.e. a trace tr, where the node $A$ starts a new session by acting as a source. Of course, if this action is the only activity of the network, there is no hope to confuse the attacker. The idea is to see whether the attacker can be confused when the traffic is sufficient. In particular, we may want to consider a situation, i.e. a trace $\mathrm{tr}^{+}$, where a request also arrives at node $A$ at the same time, so that the node $A$ has also the possibility to act as a forwarder. However, since a request conveys a unique identifier $i d$, it will be easy for the attacker to observe whether $A$ is acting as a source (the request will contain a fresh identifier) or as a forwarder (the request will contain an identifier that has been previously observed by the attacker). Actually, the same reasoning allows us to conclude that indistinguishability is not preserved w.r.t. the role Dest: a reply conveys a globally unique nonce (namely chall).

The updated version of ANODR proposed in 15] and informally described below (see the appendice for a formal description) fixes the issue regarding indistinguishability w.r.t. Roles $=\{$ Dest $\}$. In this version, $K_{T}$ is a symmetric encryption key shared between the source $A$ and the destination $D ; K_{A}, K_{B}$ and $K_{C}$ are symmetric keys known only to their owners $A, B, C$, whereas $K_{\text {seed } B}$, $K_{\text {seedC }}, K_{\text {seedD }}$ are fresh keys shared between consecutive nodes on the reply route. The key $K_{D}$ is generated by $A$ and will be known by every node on the route by the end of a session. The routes are stored as a correspondence between route pseudonyms (the $N_{i}$ ) by each intermediate node. The proof of opening takes the form of the key $K_{D}$ which is embedded in an onion which is different from the onions used during the request phase. For sake of clarity, we use $\{\cdot\}$. instead of senc and aenc, and we omit some $\langle\cdot, \cdot\rangle$.

$$
\begin{aligned}
& A \rightarrow B:\left\langle\operatorname{req}, i d, \operatorname{pub}(A),\left\{\text { dest, } K_{D}\right\}_{K_{T}},\{\text { dest }\}_{K_{D}},\{\operatorname{src}\}_{K_{A}}\right\rangle \\
& B \rightarrow C:\left\langle\operatorname{req}, i d, \operatorname{pub}(B),\left\{\text { dest, } K_{D}\right\}_{K_{T}},\{\text { dest }\}_{K_{D}},\left\{N_{B},\{\operatorname{src}\}_{K_{A}}\right\}_{K_{B}}\right\rangle \\
& C \rightarrow D:\left\langle\operatorname{req}, i d, \operatorname{pub}(C),\left\{\text { dest, } K_{D}\right\}_{K_{T}},\{\text { dest }\}_{K_{D}},\left\{N_{C},\left\{N_{B},\{\operatorname{src}\}_{K_{A}}\right\}_{K_{B}}\right\}_{K_{C}}\right\rangle \\
& D \rightarrow C:\left\langle\operatorname{rep},\left\{K_{\text {seed } D}\right\}_{\operatorname{pub}(C)},\left\{K_{D},\left\{N_{C},\left\{N_{B},\{\operatorname{src}\}_{K_{A}}\right\}_{K_{B}}\right\}_{K_{C}}\right\}_{K_{\text {seed }}}\right\rangle \\
& C \rightarrow B:\left\langle\operatorname{rep},\left\{K_{\text {seed } C}\right\}_{\operatorname{pub}(B)},\left\{K_{D},\left\{N_{B},\{\operatorname{src}\}_{K_{A}}\right\}_{K_{B}}\right\}_{K_{\text {seed }}}\right\rangle \\
& B \rightarrow A:\left\langle\operatorname{rep},\left\{K_{\text {seed } B}\right\}_{\operatorname{pub}(A)},\left\{K_{D},\{\operatorname{src}\}_{K_{A}}\right\}_{K_{\text {seed }} B}\right\rangle
\end{aligned}
$$

Considering a topology $\mathcal{T}$ such that any malicious node has at least two distinct neighbours other than itself, and an initial configuration $K_{0}$ associated to the updated version of ANODR and $\mathcal{T}$, we have that $K_{0}$ preserves indistinguishability w.r.t. Roles $=\{$ Dest $\}$, according to Definition 4 .

Intuitively, for each trace $\operatorname{tr}$ in which the node $A$ (under the scrutiny of the attacker) acts as a destination, we will consider a trace $\mathrm{tr}^{+}$which extends $\mathrm{tr}$ and 
such that the node $A$ has at least two reply to treat (one as a destination and one as a forwarder). Since the proof of opening and the onion are modified at each hop of the route, the attacker will not be able to observe whether two reply packets come from the same session or not. Thus, he can not be sure that the action of interest has been done by the role Dest.

\section{$5 \quad$ Unlinkability}

We focus here on a different kind of anonymity: the (un)ability for the attacker to determine whether two messages belong to the same session. Note that an attacker able to determine whether two reply messages belong to the same session will gain valuable information about the route being established.

\subsection{Augmented Process}

To define unlinkability, we need a notion of session. Note that, in our setting, a session may involve an arbitrary number of actions since we do not know in advance the length of the path from the source to the destination. In order to define this notion formally, we need to be able to track an execution of the process through the entire network, goal which is achieved through a notion of augmented processes. Thus, given a routing protocol $\mathcal{P}_{\text {routing, }}$, we define its augmentation $\tilde{\mathcal{P}}_{\text {routing }}$ and modify the operational semantics accordingly to trace an execution of one session of the protocol. We also add some information about the source and the destination. This information will be useful later on to define our notion of anonymity (see Section 6).

For sake of simplicity, we consider a routing protocol that is made up of parametrized processes of two different kinds. Even if these syntactic restrictions seem to be very specific, our definition actually captures most of the routing protocols and are quite natural.

Initiator: a parametrized process with two parameters $P\left(z_{S}, z_{D}\right)$ such that its first communication action is an output possibly followed by several inputs. In such a case, its augmentation $\tilde{P}\left(z_{S}, z_{D}\right)$ is obtained from $P\left(z_{S}, z_{D}\right)$ by adding the prefix new sid. to it, by replacing the action out $(u)$ with out $\left(\left\langle u,\left\langle\operatorname{sid}, z_{S}, z_{D}\right\rangle\right\rangle\right.$, and replacing each action in $(u)$ with $\operatorname{in}\left(\left\langle u,\left\langle x_{1}, x_{2}, x_{3}\right\rangle\right\rangle\right)$ where $x_{1}, x_{2}, x_{3}$ are fresh variables.

Responder: a parametrized process with one parameter $P\left(z_{V}\right)$ such that its first communication action is an input possibly followed by several outputs. In such a case, its augmentation $\tilde{P}\left(z_{V}\right)$ is obtained from $P\left(z_{V}\right)$ by replacing the action $\operatorname{in}(u)$ with $\operatorname{in}\left(\left\langle u,\left\langle x_{1}, x_{2}, x_{3}\right\rangle\right\rangle\right)$ where $x_{1}, x_{2}, x_{3}$ are fresh variables, and each action out $(u)$ with out $\left(\left\langle u,\left\langle x_{1}, x_{2}, x_{3}\right\rangle\right\rangle\right)$.

Now, to prevent the additional information that is conveyed by the messages to occur in the frame, we need to adapt our operational semantics. Basically, when we perform a communication, we only add the first projection of the outputted term in the frame. The second projection of the outputted term is added under the arrow as an annotation. 
Example 7. Back to Example 3, the counterpart of the trace tr, where only visible actions have been exhibited, is succinctly depicted below:

$$
\tilde{K}_{0} \underset{B, \operatorname{Src}, s i d, B, D}{\stackrel{\operatorname{out}\left(y_{1}\right), B}{\longrightarrow}} \tilde{K}_{1} \underset{C, \operatorname{Req}, \text { sid }, B, D}{\stackrel{\operatorname{out}\left(y_{2}\right), C}{\longrightarrow}} \tilde{K}_{2}
$$

where the configurations $\tilde{K}_{0}, \tilde{K}_{1}$ and $\tilde{K}_{2}$ are the counterpart of $K_{1}, K_{2}$, and $K_{3}$. The annotations under the arrows witness the fact that the two messages come from the same session sid which was initiated by $B$ to obtain a route towards $D$.

Note that only observable action will benefit from this annotation. For sake of simplicity, we write $K \underset{A, \mathrm{R},[\operatorname{sid}, S, D]}{\stackrel{\ell}{\longrightarrow}} K^{\prime}$ even in presence of an unobservable action $\ell$ (i.e. when $\ell=\tau$ ) and we add the brackets to emphasize the fact that $[\operatorname{sid}, S, D]$ is optional. Actually, the annotation is undefined in this case.

\section{$5.2 \quad$ Formalising Unlinkability}

Intuitively, unlinkability means that an attacker cannot tell whether two visible actions of a trace tr belong to the same session. As it was the case for indistinguishability, one cannot expect to achieve this goal without any sufficient traffic on the network. Moreover, due to the globally unique identifier that occur for efficiency purposes in many routing protocols (e.g. the nonce id in ANODR), there is no hope to achieve unlinkability for request messages. However, this is not a big issue since these messages are flooded in the network and thus tracking them is useless. We may want to study unlinkability for particular sets of roles, and our definition allows one to do that.

Definition 5 (unlinkability). Let $K_{0}$ be an initial configuration associated to a routing protocol and a topology, and Roles $_{1}$, Roles 2 be two sets of roles. We say that $K_{0}$ preserves unlinkability w.r.t. Roles ${ }_{1} /$ Roles $_{2}$ if for any annotated trace $\mathrm{tr}$

$$
\operatorname{tr}=K_{0} \underset{A_{1}, \mathrm{R}_{1},\left[s i d_{1}, S_{1}, D_{1}\right]}{\longrightarrow} K_{1} \underset{A_{2}, \mathrm{R}_{2},\left[s i d_{2}, S_{2}, D_{2}\right]}{\longrightarrow} \cdots \frac{\ell_{n}}{A_{n}, \mathrm{R}_{n},\left[s i d_{n}, S_{n}, D_{n}\right]} K_{n}
$$

and for any $i, j \in\{1, \ldots, n\}$ such that $\mathrm{R}_{i} \in$ Roles $_{1}, \mathrm{R}_{j} \in$ Roles $_{2}$, sid $_{i}=$ sid $_{j}$, and $\ell_{i}, \ell_{j} \neq \tau$ (i.e. $\ell_{i}, \ell_{j}$ are actions observed by the attacker), there exist two

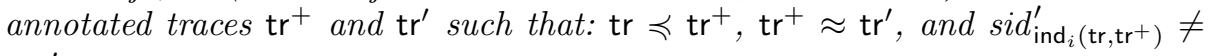
sid $_{\text {ind }_{j}\left(\mathrm{tr}, \mathrm{tr}^{+}\right)}^{\prime}$ where

$$
\operatorname{tr}^{\prime}=K_{0}^{\prime} \underset{A_{1}^{\prime}, \mathrm{R}_{1}^{\prime},\left[s i d_{1}^{\prime}, S_{1}^{\prime}, D_{1}^{\prime}\right]}{\longrightarrow} K_{1}^{\prime} \underset{\ell_{2}^{\prime}, \mathrm{R}_{2}^{\prime},\left[s i d_{2}^{\prime}, S_{2}^{\prime}, D_{2}^{\prime}\right]}{\longrightarrow} \ldots \frac{\ell_{n^{\prime}}^{\prime}}{\underset{A_{n^{\prime}}^{\prime}, \mathrm{R}_{n^{\prime}}^{\prime}, s i d_{n^{\prime}}^{\prime}, S_{n^{\prime}}^{\prime}, D_{n^{\prime}}^{\prime}}{\longrightarrow}} K_{n^{\prime}}^{\prime}
$$

Unlinkability versus indistinguishability. Note that unlinkability is a distinct notion from the indistinguishability notion exposed in Section 4. Protocols unlinkable w.r.t. any reasonable topology can be designed so as not to be indistinguishable for any role. An example of such a protocol would be $\mathcal{P}=$ $\left\{P_{1}\left(z_{S}, z_{D}\right), P_{2}\left(z_{V}\right)\right\}$ defined as follows: 


$$
\left.P_{1}\left(z_{S}, z_{D}\right)=\operatorname{out}(\operatorname{src}) \cdot \operatorname{in}(x) \quad P_{2}\left(z_{V}\right)=\operatorname{in}(x) \text {.out(dest }\right)
$$

where src and dest are two constants. The unlinkability is a consequence of emitting the same messages for every session, whereas the indistinguishability fails as the constant src (resp. dest) identifies the role $P_{1}$ (resp. $P_{2}$ ).

Reciprocally one can design protocols preserving indistinguishability for certain roles but not unlinkability for any two subsets of roles. The protocol $\mathcal{P}^{\prime}$ made up of the three roles described below fails clearly at preserving the unlinkability w.r.t. any non-trivial topology for any sets of roles Roles 1 and Roles 2 as it mimicks the session identifiers introduced formerly.

$$
\begin{gathered}
P_{1}^{\prime}\left(z_{S}, z_{D}\right)=\text { new } n \text {.out }(n) \cdot \operatorname{in}(x) \quad P_{2}^{\prime}\left(z_{V}\right)=\operatorname{in}(x) \cdot \operatorname{out}(x) \\
P_{3}^{\prime}\left(z_{V}\right)=\operatorname{in}(x) \cdot \operatorname{store}(x) \cdot \operatorname{out}(x)
\end{gathered}
$$

On the other hand, the indistinguishability w.r.t. any topology for either $P_{2}^{\prime}$ or $P_{3}^{\prime}$ is trivially preserved as the roles are essentially the same.

\subsection{Analysis of ANODR}

As discussed at the beginning of Section 5.2. ANODR, as many other routing protocols, does not preserve unlinkability (as soon as the underlying topology is non-trivial topology) for sets Roles $1=$ Roles $_{2}=\{$ Src, Req $\}$ due to the forwarding of the same $i d$ by every intermediate node during the request phase. Actually, the simplified version of ANODR presented in Section 3.2 does not preserve unlinkability for sets Roles $_{1}=$ Roles $_{2}=\{$ Dest, Rep $\}$ due to the forwarding of the nonce chall by every intermediate node during the reply phase. This version does not preserve unlinkability for sets $\{$ Src, Req $\} /\{$ Dest, Rep $\}$ either. Indeed, during the request phase, the nodes will emit a message containing an onion, and during the reply phase, they are waiting for a message that contains exactly the same onion. This allows the attacker to link a request message with a reply message and to identify them as coming from the same session.

The updated version of ANODR (see Section 4.2) actually fixes the two last issues. Again, we need for this to consider topologies $\mathcal{T}$ for which any malicious node has at least two distinct neighbours other than itself. In such a situation, following the same ideas as the one used to establish indistinguishability, we can show that an initial configuation $K_{0}$ preserves unlinkability w.r.t. $\{$ Dest, Rep\}/ $\{$ Dest, $\operatorname{Rep}\}$, and $\{$ Src, Req $\} /\{$ Dest, Rep $\}$ (according to Definition 5).

\section{Anonymity}

Anonymity is concerned with hiding who performed a given action. Here, we are not concerned by hiding the identity of the sender (or the receiver) of a given message, but we would like to hide the identity of the source (or the destination) of the request/reply message. When the identity of the source is hidden, we speak about source anonymity. Similarly, when the identity of the destination is hidden, we speak about destination anonymity. Again, we consider both types 
of anonymity with respect to an external eavesdropper that is localised to some nodes (possibly every one of them) of the network.

As in Section 5, to define the anonymity, we need to link messages occurring at various places in the network to their respective source and destination, thus we consider the augmented version of the protocol as in Section 5.1

\subsection{Formalising Anonymity}

Intuitively, source (resp. destination) anonymity can be achieved if the attacker is unable to tell the source (resp. the destination) of an observed message. This idea can actually be interpreted as the existence of anonymity sets of cardinal greater or equal than two. As for the previous privacy-type notions, one cannot expect to hide the source (resp. destination) of an action in a trace tr without any sufficient traffic as it would be easy for an attacker to observe the first node to output a request (resp. a reply) and deduce the source (resp. destination) of this execution. For this reason, anonymity will be achieved if there exist two other traces $\mathrm{tr}^{+}$and $\mathrm{tr}^{\prime}$ of the system which look the same to the attacker, and in which the corresponding transitions have different sources (resp. destinations).

Definition 6 (anonymity). Let $K_{0}$ be an initial configuration associated to a routing protocol and a topology. We say that $K_{0}$ preserves source anonymity (resp. destination anonymity) if for any annotated trace $\mathrm{tr}$

$$
\operatorname{tr}=K_{0} \underset{A_{1}, \mathrm{R}_{1},\left[\operatorname{sid}_{1}, S_{1}, D_{1}\right]}{\longrightarrow} K_{1} \underset{A_{2}, \mathrm{R}_{2},\left[\operatorname{sid}_{2}, S_{2}, D_{2}\right]}{\longrightarrow} \ldots \frac{\ell_{n}}{A_{n}, \mathrm{R}_{n},\left[\operatorname{sid}_{n}, S_{n}, D_{n}\right]} K_{n}
$$

and for any $i \in\{1, \ldots, n\}$ such that $\ell_{i} \neq \tau$ (i.e. $\ell_{i}$ is an action observed by the attacker), there exist two annotated traces $\mathrm{tr}^{+}$and $\mathrm{tr}^{\prime}$ such that $\mathrm{tr} \preccurlyeq \mathrm{tr}^{+}$,

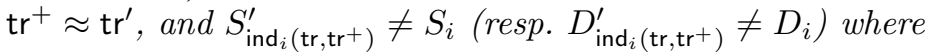

$$
\operatorname{tr}^{\prime}=K_{0}^{\prime} \underset{A_{1}^{\prime}, \mathrm{R}_{1}^{\prime},\left[s i d_{1}^{\prime}, S_{1}^{\prime}, D_{1}^{\prime}\right]}{\longrightarrow} K_{1}^{\prime} \underset{\ell_{2}^{\prime}, \mathrm{R}_{2}^{\prime},\left[\operatorname{sid}_{2}^{\prime}, S_{2}^{\prime}, D_{2}^{\prime}\right]}{\longrightarrow} \cdots \frac{\ell_{n^{\prime}}^{\prime}}{\underset{A_{n^{\prime}}^{\prime}, \mathrm{R}_{n^{\prime}}^{\prime},\left[\operatorname{sid}_{n^{\prime}}^{\prime}, S_{n^{\prime}}^{\prime}, D_{n^{\prime}}^{\prime}\right]}{\longrightarrow}} K_{n^{\prime}}^{\prime}
$$

\subsection{Anonymity versus Indistinguishability/Unlinkability}

The notions of source and destination anonymity are distinct from indistinguishability for a set of roles and unlinkability of two sets of roles. The protocol $\mathcal{P}=\left\{P_{1}\left(z_{S}, z_{D}\right), P_{2}\left(z_{V}\right)\right\}$ where $P_{1}\left(z_{S}, z_{D}\right)=\operatorname{out}\left(z_{S}\right) \cdot \operatorname{in}(x)$, and $P_{2}\left(z_{V}\right)=$ in $(x)$.out $(x)$ preserves both the indistinguishability of $P_{1}$ (a node can play $P_{2}$ as a response to a session it initiated previously as $P_{1}$ ) and the unlinkability of any two subsets of $\left\{P_{1}, P_{2}\right\}$ (as every session with the same node as a source will generate the exact same messages) but not source anonymity as the identity of the source is obvious for any attacker along the route. A symmetrical protocol can be built by replacing $z_{S}$ with $z_{D}$ in $P_{1}$ to disclose the destination of a session without breaking the indistinguishability.

Conversely, the protocol $\mathcal{P}=\left\{P_{1}\left(z_{S}, z_{D}\right), P_{2}\left(z_{V}\right)\right\}$ defined as

$$
P_{1}\left(z_{S}, z_{D}\right)=\text { new } n \text {.out }(\langle\operatorname{src}, n\rangle) \cdot \operatorname{in}(x) \quad P_{2}\left(z_{V}\right)=\operatorname{in}(\langle x, y\rangle) \text {.out }(\langle\text { dest }, y\rangle)
$$


preserves destination anonymity as any node can play $P_{2}$ in response to a request, whatever the original destination was. Indeed, given such a topology $\mathcal{T}$, a trace tr of the protocol, and a visible action $\ell_{i}=(\operatorname{out}(y), A)$ associated to a a source $S_{i}=S$ and a destination $D_{i}=A$, we can let $\operatorname{tr}^{+}$be equal to $\operatorname{tr}$ and define $\operatorname{tr}^{\prime}$ to be the trace mimicking $\operatorname{tr}$ but with $S$ as the source and destination of the request associated to $\ell_{i}$. The equivalence of $\operatorname{tr}$ and $\operatorname{tr}^{\prime}$ comes from the content of their frames which is limited to the names of the request sources, identical in both cases. On the other hand, $\mathcal{P}$ does not preserve indistinguishability of $P_{1}$ or $P_{2}$, nor unlinkability of any two subsets of $\left\{P_{1}, P_{2}\right\}$ as session identifiers and constants to distinguish roles are embedded in the protocol.

However, intuitively, there is a relation between source anonymity (resp. destination anonymity) and indistinguishability of the role source (resp. destination). Indeed, source anonymity seems to imply that the action of interest can be mimicked by someone different from source, and thus who should not play the role source. Thus, restricting ourselves to "reasonable" routing protocols, we are indeed able to establish this relation. For this, we define source and destination roles as roles which are only used by nodes acting as sources or destinations.

Definition 7 (acting as a source (resp. destination)). Let $K_{0}$ be an initial configuration associated to a routing protocol and a topology. We say that Roles is the set of roles acting as a source (resp. acting as a destination) if for any annotated trace $\operatorname{tr}$ with $\ell_{1}, \ldots, \ell_{n} \neq \tau$

$$
\operatorname{tr}=K_{0} \underset{A_{1}, \mathrm{R}_{1}, \text { sid }_{1}, S_{1}, D_{1}}{\Longrightarrow} K_{1} \underset{\ell_{2}, \mathrm{\ell}_{2}, \text { sid }, S_{2}, D_{2}}{\Longrightarrow} \cdots \frac{\ell_{n}}{\underset{A_{n}, \mathrm{R}_{n}, \text { sid }_{n}, S_{n}, D_{n}}{\Longrightarrow}} K_{n}
$$

and for any $i \in\{1, \ldots, n\}, \mathrm{R}_{i} \in$ Roles if and only if $A_{i}=S_{i}$ (resp. if and only if $A_{i}=D_{i}$ ).

In case of ANODR (both versions), the set of roles acting as a source is $\{\mathrm{Src}\}$. This is the only role able to spontaneously start a session and it is unable to reply to a request. The set of roles acting as a destination is limited to $\{$ Dest $\}$. The proof of opening prevents any node other than the destination to play it and, conversely, a destination node can only play the role Dest as a response to such a request. Note that, for some badly designed routing protocols, it may happen that the set of roles acting as a source (resp. destination) is empty. In such a case, the proposition below is trivially true.

Proposition 2. Let $K_{0}$ be an initial configuration associated to a routing protocol and a topology. If $K_{0}$ preserves source (resp. destination) anonymity, then it preserves indistinguishability w.r.t. the set of roles acting as a source (resp. destination).

\subsection{Analysis of ANODR}

In this section, we apply our formalisation of anonymity to the ANODR routing protocol. As a consequence of Propositions 1 1 and 2, we have the following result. 
Corollary 1. Let $\mathcal{T}$ be a topology with a malicious node that has only malicious neighbours, and $K_{0}$ be an initial configuration associated to $\mathcal{P}_{A N O D R}^{\mathrm{simp}}$ and $\mathcal{T}$. We have that $K_{0}$ preserves neither source nor destination anonymity.

For the updated version of ANODR, similarly, we can show that it does not preserve source anonymity. However, this protocol seems to have been designed to achieve destination anonymity. Indeed, considering topologies for which any malicious node has at least one neighbour other than itself, we can show that any trace $\operatorname{tr}$ can be extended to $\mathrm{tr}^{+}$so that the node of interest has at least two reply to treat (one as the destination of the request, and the other one as the forwarder). This is actually sufficient to confuse the attacker who observes the network, and to establish anonymity of the destination according to Definition 6 .

\section{Conclusion}

We have defined a framework for modeling routing protocols in ad hoc networks in a variant of the applied pi-calculus. Within this framework we can stipulate which agents are subject to the attention of a global eavesdropper. We were able to propose several definitions for privacy-type properties that encompass the specificity of a given network topology. We illustrate these definitions on the anonymous routing protocol ANODR, considered in two versions, and thus provide a partial formal security analysis of its anonymity.

As future work, it would be interesting to have a more general model of protocols to represent high-level operations in routing protocols (e.g. reversing a list). However, since our definitions are expressed in terms of traces, this should not impact so much the privacy definitions proposed in this paper. Another direction is the enrichment of our attacker model, so as to model fully compromised nodes which disclose their long-term keys or fresh nonces generated during the execution of the protocols, and active attackers able to forge messages and interact with honest agents. Finally, from the point of view of the verification, a reduction result on network topologies as presented in [11] would make the perspective of automated proofs of anonymity easier.

\section{References}

1. Abadi, M., Fournet, C.: Mobile values, new names, and secure communication. In: Proc. 28th Symposium on Principles of Programming Languages, POPL 2001, pp. 104-115. ACM Press (2001)

2. Abadi, M., Gordon, A.: A calculus for cryptographic protocols: The spi calculus. In: Proc. 4th Conference on Computer and Communications Security, CCS 1997, pp. 36-47. ACM Press (1997)

3. Arapinis, M., Chothia, T., Ritter, E., Ryan, M.: Analysing unlinkability and anonymity using the applied pi calculus. In: Proc. 23rd Computer Security Foundations Symposium, CSF 2010, pp. 107-121. IEEE Computer Society Press (2010)

4. Armando, A., Carbone, R., Compagna, L., Cuéllar, J., Tobarra, M.L.: Formal analysis of SAML 2.0 web browser single sign-on: breaking the SAML-based single sign-on for google apps. In: Proc. of the 6th ACM Workshop on Formal Methods in Security Engineering, FMSE 2008, pp. 1-10. ACM (2008) 
5. Armando, A., Basin, D., Boichut, Y., Chevalier, Y., Compagna, L., Cuellar, J., Hankes Drielsma, P., Heám, P.C., Kouchnarenko, O., Mantovani, J., Mödersheim, S., von Oheimb, D., Rusinowitch, M., Santiago, J., Turuani, M., Viganò, L., Vigneron, L.: The AVISPA Tool for the Automated Validation of Internet Security Protocols and Applications. In: Etessami, K., Rajamani, S.K. (eds.) CAV 2005. LNCS, vol. 3576, pp. 281-285. Springer, Heidelberg (2005)

6. Arnaud, M., Cortier, V., Delaune, S.: Modeling and verifying ad hoc routing protocols. In: Proc. 23rd IEEE Computer Security Foundations Symposium, CSF 2010, pp. 59-74. IEEE Computer Society Press (July 2010)

7. Backes, M., Hritcu, C., Maffei, M.: Automated verification of remote electronic voting protocols in the applied pi-calculus. IEEE Comp. Soc. Press (2008)

8. Blanchet, B.: An efficient cryptographic protocol verifier based on prolog rules. In: Proc. 14th Computer Security Foundations Workshop, CSFW 2001. IEEE Comp. Soc. Press (2001)

9. Bruso, M., Chatzikokolakis, K., den Hartog, J.: Formal verification of privacy for RFID systems. In: Proc. 23rd Computer Security Foundations Symposium, CSF 2010, IEEE Computer Society Press (2010)

10. Chrétien, R., Delaune, S.: Formal analysis of privacy for routing protocols in mobile ad hoc networks. Research Report LSV-12-21, Laboratoire Spécification et Vérification, ENS Cachan, France, 24 pages (December 2012)

11. Cortier, V., Degrieck, J., Delaune, S.: Analysing Routing Protocols: Four Nodes Topologies Are Sufficient. In: Degano, P., Guttman, J.D. (eds.) Principles of Security and Trust. LNCS, vol. 7215, pp. 30-50. Springer, Heidelberg (2012)

12. Delaune, S., Kremer, S., Ryan, M.D.: Verifying privacy-type properties of electronic voting protocols. Journal of Computer Security (4), 435-487 (2008)

13. Garcia, F.D., Hasuo, I., Pieters, W., van Rossum, P.: Provable anonymity. In: Proc. ACM Workshop on Formal Methods in Security Engineering, FMSE 2005, pp. 63-72. ACM (2005)

14. Hu, Y.-C., Perrig, A., Johnson, D.: Ariadne: A Secure On-Demand Routing Protocol for Ad Hoc Networks. Wireless Networks 11, 21-38 (2005)

15. Kong, J., Hong, X.: ANODR: anonymous on demand routing with untraceable routes for mobile ad-hoc networks. In: Proc. 4th ACM Interational Symposium on Mobile Ad Hoc Networking and Computing, MobiHoc 2003. ACM (2003)

16. Mauw, S., Verschuren, J.H.S., de Vink, E.P.: A Formalization of Anonymity and Onion Routing. In: Samarati, P., Ryan, P.Y.A., Gollmann, D., Molva, R. (eds.) ESORICS 2004. LNCS, vol. 3193, pp. 109-124. Springer, Heidelberg (2004)

17. Nanz, S., Hankin, C.: A Framework for Security Analysis of Mobile Wireless Networks. Theoretical Computer Science 367(1), 203-227 (2006)

18. Papadimitratos, P., Haas, Z.: Secure routing for mobile ad hoc networks. In: Proc. SCS Communication Networks and Distributed Systems Modelling Simulation Conference, CNDS (2002)

19. Serjantov, A., Danezis, G.: Towards an Information Theoretic Metric for Anonymity. In: Dingledine, R., Syverson, P.F. (eds.) PET 2002. LNCS, vol. 2482, pp. 41-53. Springer, Heidelberg (2003)

20. Song, R., Korba, L., Lee, G.: AnonDSR: Efficient anonymous dynamic source routing for mobile ad-hoc networks. In: Proc. ACM Workshop on Security of Ad Hoc and Sensor Networks, SASN 2005. ACM (2005)

21. Zapata, M.G., Asokan, N.: Securing ad hoc routing protocols. In: Proc. 1st ACM Workshop on Wireless SEcurity, WiSE 2002, pp. 1-10. ACM (2002) 